\title{
Femtosecond Acoustics and Terahertz Ultrasonics
}

\author{
Chi-Kuang Sun
}

Graduate Institute of Photonics and Optoelectronics, National Taiwan University, Taipei, Taiwan, $\underline{\text { sun@ @ntu.edu.tw }}$

Acoustic wave is one of the most natural tools for communication and imaging. It is used for clinical imaging, to noninvasively probe physical structures under the surface as well as thermal dynamics of objects. However, considering the bandwidth $(\mathrm{GHz})$ of current ultrasonic technologies, the electrically generated acoustic wave has a pulse duration much longer than $1 \mathrm{~ns}$ and a wavelength longer than $10 \mu \mathrm{m}$, which is far from adequate for molecular-level imaging, not to mention the femtosecond dynamics of many interacted molecules, including liquid water. Besides, it is believed that a wave-particle transition of $\mathrm{THz}$ acoustic waves must come into play when they are interacting with thermal phonons in all kinds of materials, including thin films, bulk materials, and nanostructures. To resolve the mysterious thermal dynamics in amorphous materials as well as the energy (heat) transport and dissipation from solids into liquids, an ultra-broadband $\mathrm{THz}$ acoustic source is thus urgently needed.

In this presentation, I would like to present the result of a new research direction: femtosecond acoustics and terahertz ultrasonics. The physical principle to convert a femtosecond optical pulse into a femtosecond acoustic pulse will be introduced. With a short pulse width, the spectral bandwidth can thus exceed THz. Combined with the ultraslow sound velocity, the application of this new tool is two folds. One is for $\mathrm{THz}$ phonon spectroscopy, which is critical to resolve the heat transport mysteries. The other is for in situ noninvasive sub-surface atomic level resolution imaging, which was not possible before. Examples will be given in this presentation.

Since it's proposed by H. Maris 30 years ago, "picosecond ultrasonic" has been developed as a complete technique able to study various acoustic phenomena and parameters from thickness measurements [1] to hypersound attenuation [2,3]. Based on the use of an ultrafast laser, this technique allows the generation and detection of coherent acoustic phonons (CAP) in the sub-THz range. Even though picosecond ultrasonic has been developed for more than 30 years, its frequency range can only reach $400 \mathrm{GHz}$, which is still far from THz. In 1999 , by femtosecond laser excitation of piezoelectric semiconductor quantum wells, we discovered a simple way to generate giant coherent acoustic phonons (acoustic waves) with a frequency over 2 $\mathrm{THz}$ [4]. This piezoelectric nano-layer based technique was later-on termed "nano-ultrasonics," with a focus to emphasize its capability to provide nano-meter spatial resolution ultrasonic images [5]. After over one decade's study, we have made this technique a powerful tool to image and characterize nanoscale materials and interfaces. In recent years, this method has not only been successfully applied to characterize the ångström-level surface and interface roughness [6], but also the acoustic attenuation up to $650 \mathrm{GHz}$ in amorphous $\mathrm{SiO}_{2}$ and more recently we showed the possibility to measure the acoustic attenuation spectrum in ice up to $1 \mathrm{THz}$, thus providing critical thermal dynamics parameters [7].

During my presentation, I will show the effort of the current program to not only continuously apply the $\mathrm{THz}$ acoustic spectroscopy 1). for the investigation of the Boson peak issue in amorphous solids and 2). of the collisionless decay of the coherent phonons in crystals, but also 3 ). to work on the atomic resolution imaging in solid-liquid water interface.

Amorphous material systems have impacted human lives in numerous aspects. Glasses, polymers, and even bio-tissues are significant matters made with amorphous structures. Despite the long appearance, the physical natures of the amorphous material system are still far from well understood. Unlike crystalline solids, amorphous materials exhibit a number of anomalous thermal properties. These anomalies were first discovered half a century ago by Berman, who noticed the unexpected instance of the thermal conductivity $\kappa(T)$ anomaly for vitreous silica $\left(v-\mathrm{SiO}_{2}\right)$. He measured the thermal conductivities of several $v$ $\mathrm{SiO}_{2}$ samples in the temperature range between 2.2 and $90 \mathrm{~K}$ and found the plateau behavior of thermal conductivities at around $10 \mathrm{~K}$, corresponding to a phonon peak frequency of $\sim 1 \mathrm{THz}$, with a magnitude several orders smaller. Since then, several other techniques, such as Raman scattering, inelastic neutron scattering, and X-ray scattering, had been carried out to explore this anomalous thermal phenomenon. Although many had witnessed the anomaly, none of them were able to provide a comprehensive understanding of its origin.

In crystals, the low-frequency density of states (DOS), $g(\omega)$, follows the so-called Debye model well; that is, by simply considering possible modes of sound waves which match the boundary conditions, one obtains the square frequency dependence of the DOS, $g(\omega) \propto \omega^{2}$. In contrast, amorphous material systems show an excess contribution to the DOS at the $\mathrm{THz}$ frequency regime (or at $\sim 5 \mathrm{meV}$ energy level appeared in inelastic scattering spectra), which in turn results in a decreased thermal conductivity or an increased specific heat, as evidenced by previous vitreous silica experimental results. The excess in DOS is usually identified as a peak in a reduced DOS representation, $g(\omega) / \omega^{2}$ vs $\omega$. At the time of its discovery, little was known about this feature except for its bosonic character. Therefore, it became known as the "boson peak" (BP). The physical origin as well as dynamics of the Boson Peak remained puzzling and controversial due to the fact that no acoustic 
attenuation spectral measurement in this frequency range can be directly performed in amorphous materials to verify the correctness of different theoretical models.

In contrast to amorphous solids, in past decades, hypersound wave propagation in crystalline solids have drawn much attention in a wide variety of scientific studies focusing on topics such as the spontaneous decay of longitudinal acoustic phonons, picosecond ultrasonics, nanoultrasonics, and the development of acoustic phonon lasers. In the frequency range of $0.4-3 \mathrm{THz}$, coherent acoustic phonons (or hypersound waves) in crystal are physically modeled to be attenuated due to collisions with thermal phonons. However, different theories were debated with no experimental proof to distinguish their correctness and appropriate frequency ranges.

In crystalline dielectrics, this hypersound propagation loss can arise from both extrinsic and intrinsic mechanisms. The extrinsic mechanisms are commonly dominated by phonon-scattering processes at lattice imperfections. When these extrinsic mechanisms are absent, the acoustic loss will ultimately be determined by intrinsic anharmonic decay. Based on low frequency data, the mostly accepted picture is as follow. In most solids, thermal phonons have a lifetime, $\tau$, on the order of picosecond to femtosecond. When the angular frequency, $\omega$, of the acoustic waves is small compared to the thermal phonon lifetime with $\omega \tau<<1$ (usually in the sub-THz range), the propagation of acoustic waves will drive the thermal phonons out of equilibrium, and causing the interaction and resulting in the acoustic wave attenuation. Due to the fact that the acoustic waves are with a wavelength much longer than most roomtemperature thermal phonons, the propagating coherent phonons will be scattered by all different thermal phonons and the momentum conservation is not a concern, due to the large uncertainty. This model well describes all lower-frequency (below $400 \mathrm{GHz}$ ) measurement on different solids.

However it has been long debated and postulated that, when $\omega \tau \gg>1$ (usually above $\mathrm{THz}$ at room temperature), acoustic waves have to be considered as coherent phonons and the attenuation of coherent phonons due to their interaction with thermal phonons will be limited due to specific selection rules for energy and momentum conservation. That is to say that, only selected thermal phonons with specific momentums and energies involve in this "3-phonon interaction" process, thus making $\mathrm{THz}$ acoustic wave attenuation "saturated." In the transition range between these two regimes, $\omega \tau \gg>1$, the acoustic frequency will be around $\mathrm{THz}$ for room temperature crystals.

In this frequency range, the coherent acoustic phonon attenuation processes have never been explored and have been under debate for almost four decades due to the lack of reliable $\mathrm{THz}$ acoustic transducers. Applying our developed $\mathrm{THz}$ nanoultrasound technique and studying the frequency dependent behavior of coherent acoustic phonons in this ambiguous transition range is therefore crucial since it will bring critical understanding for thermal properties in solids.

For nanoimaging with a femtosecond acoustic pulse, one can take advantage of the ultraslow sound velocity, which is usually on the order of $3000 \mathrm{~m} / \mathrm{s}$ and the ultrahigh temporal resolution which is usually on the order of $100 \mathrm{fs}$. It can be noted that within 100 fs, a femtosecond acoustic pulse travels only a distance of 3 angstroms, assuming a $3000 \mathrm{~m} / \mathrm{s}$ velocity. Femtosecond time-resolved sound propagation imaging can thus provide a spatial resolution down to 1.5 angstroms assuming an acoustic round trip path. To study the performance of femtosecond acoustics applied on in situ monitoring a chemical reaction at a solid/liquid interface, the well-known anode-oxidation process occurring during the GaN-based photoelectro-chemical (PEC) water splitting was taken as our model. As a result, sub-atomic resolution imaging was observed in situ noninvasively [8].

This project is sponsored by Ministry of Science and Technology of Taiwan under 106-2112-M-002 004 -MY3 and National Taiwan University under $107 \mathrm{~L} 880404$.

\section{References}

1. Thomsen, C., Grahn, H. T., Maris H. J., Tauc, J. Surface generation and detection of phonons by picosecond light pulses // Phys. Rev. B, 1986. V. 34, No. 6, P. 41294138 .

2. Lin, H. N., Stoner, R. J., Maris, H. J., Tauc, J. Phonon attenuation and velocity measurements in transparent materials by picosecond acoustic interferometry // J. Appl. Phys., 1991. V. 69, No. 7. P. 3816-3822.

3. Duquesne, J.-Y., Perrin, B. Ultrasonic attenuation in a quasicrystal studied by picosecond acoustics as a function of temperature and frequency // Phys. Rev. B, 2003. V. 68, No. 13. 134205.

4. Sun, C. K., Liang, J. C., $Y u, X Y$. Coherent acoustic phonon oscillations in semiconductor multiple-quantumwells with piezoelectric fields // Physical Review Letters 2000. V. 84, No. 1, P. 179-182.

5. Lin, K.-H., Lai, C.-M., Pan, C.-C., Chyi, J.-I., Shi, J.W., Sun, S.-Z., Chang, C.-F., Sun, C.-K. Spatial manipulation of nanoacoustic waves with a nanoscale spot size // Nature Nanotechnology 2007. V. 2, No. 11, P. 704708 .

6. Wen, Y.-C., Hsieh, C.-L., Lin, K.-H., Chen, H.-P., Chin, S.-C., Hsiao, C.-L., Lin, Y.-T., Chang, C.-S., Chang, Y.-C., Tu, L.-W., Sun, C.-K. Specular scattering probability of acoustic phonons in atomically flat interfaces // Physical Review Letters 2009. V. 103, No. 26. 264301.

7. Mante, P.-A., Chen, C.-C., Wen, Y.-C., Sheu, J.-K., Sun, $C .-K$. Thermal boundary resistance between $\mathrm{GaN}$ and cubic ice and $\mathrm{THz}$ acoustic attenuation of cubic ice from complex acoustic impedance measurements // Physical Review Letters 2013. V. 111, No. 22. 225901.

8. Shen, C.-C., Weng, M.-Y., Sheu, J.-K., Yao, Y.-T., Sun, $C$. $-K$. In situ monitoring of chemical reactions at a solid-water interface by femtosecond acoustics // Journal of Physical Chemistry Letters 2017. V. 8, No. 21. P. 54305437. 